\title{
Gamma tocotrienol targets tyrosine phosphatase SHP2 in mammospheres resulting in cell death through RAS/ERK pathway
}

\author{
Wenyi Gu*, Indira Prasadam², Meihua Yu ${ }^{1}$ Fengxia Zhang ${ }^{3}$, Patrick Ling ${ }^{2}$, Yin Xiao ${ }^{2}$ and Chengzhong Yu ${ }^{1 *}$
}

\begin{abstract}
Background: There is increasing evidence supporting the concept of cancer stem cells (CSCs), which are responsible for the initiation, growth and metastasis of tumors. CSCs are thus considered the target for future cancer therapies. To achieve this goal, identifying potential therapeutic targets for CSCs is essential.

Methods: We used a natural product of vitamin E, gamma tocotrienol (gamma-T3), to treat mammospheres and spheres from colon and cervical cancers. Western blotting and real-time RT-PCR were employed to identify the gene and protein targets of gamma-T3 in mammospheres.

Results: We found that mammosphere growth was inhibited in a dose dependent manner, with total inhibition at high doses. Gamma-T3 also inhibited sphere growth in two other human epithelial cancers, colon and cervix. Our results suggested that both Src homology 2 domain-containing phosphatase 1 (SHP1) and 2 (SHP2) were affected by gamma-T3 which was accompanied by a decrease in $\mathrm{K}$ - and $\mathrm{H}$-Ras gene expression and phosphorylated ERK protein levels in a dose dependent way. In contrast, expression of self-renewal genes TGF-beta and LIF, as well as ESR signal pathways were not affected by the treatment. These results suggest that gamma-T3 specifically targets SHP2 and the RAS/ERK signaling pathway.
\end{abstract}

Conclusions: SHP1 and SHP2 are potential therapeutic targets for breast CSCS and gamma-T3 is a promising natural drug for future breast cancer therapy.

\section{Background}

The concept of cancer stem cells (CSCs) describes that tumors contain a small proportion of self-renewing and pluri-potent cells that are responsible for initiating and maintaining tumor growth [1]. This concept is well established in leukemia and has also been reported in a few solid tumor types [2-4]. Recent studies further confirm that a specific cell population is responsible for the initiation and growth of solid tumors [5-7]. These cells usually express high levels of multiple drug resistant gene (MDR1) [8] and ATP binding cassette (ABC) transporter [9] and are therefore resistant to chemotherapy and considered as the major source of drug-resistance in tumors. Moreover, it has been demonstrated that CSCs

\footnotetext{
* Correspondence: w.gu@uq.edu.au; c.yu@uq.eud.au

'Australian Institute for Bioengineering and Nanotechnology, the University of Queensland, The corner of Cooper Rd. St Lucia, Brisbane QLD 4072, Australia

Full list of author information is available at the end of the article
}

are responsible for metastasis $[7,10,11]$, which is another major cause of cancer-related death. CSCs are thus regarded as an essential target for future advanced cancer therapy.

To achieve the goal of effective treatment of CSCs, identifying specific therapeutic targets is vital. Apart from high throughput screening methods such as microarrays, identifying novel targets of inhibitors or natural drugs is an alternative. A few natural compounds are reported to have inhibitory effects on CSCs [12-14]. These products are valuable for future CSC targeted therapy as they are normally less toxic than chemotherapeutic drugs. For example, vitamin E isotype gamma tocotrienol $(\gamma-\mathrm{T} 3)$ was shown to be effective at inhibiting cancer cell growth in several solid tumor models through apoptosis or cell stress related pathways [15-18]. In CSCs, Ling and colleagues reported that $\gamma$-T3 could effectively inhibit CSC growth in prostate cancer in vitro and in vivo [19]. They also showed that the CD44 expression of the CSCs was decreased by 
$\gamma$-T3 treatment. CD44 is one of the important epithelial CSC markers, suggesting $\gamma$-T3 may affect the stemness of prostate CSCs. However, the detailed mechanism of how $\gamma$-T3 suppresses CD44 expression and prostate CSC growth remains unknown. In addition, it is still not clear whether the reduction of CD44 expression was through $\gamma$ T3 directly interacting with CD44 or through an indirect interaction with other molecules.

Previously, a study reported that $\gamma$-T3 inhibited STAT3 phosphorylation and JAK/STAT pathway activation in different melanoma cell lines, resulting in apoptosis of the cancer cells [20]. This inhibition was through the induction of SHP1 expression by $\gamma-\mathrm{T} 3$, suggesting that SHP1 was a target of $\gamma$-T3 [20]. However, whether this is the case in CSCs has not been reported or whether there are any new targets in JAK/Stat pathway for $\gamma$-T3 remains unknown. In JAK/Stat pathway, there are two very closely related proteins SHP1 and SHP2, they share highly similar structures and sequences. Both of them have two Src homology 2 domains ( $\mathrm{SH} 2)$ that bind to several tyrosinephosphorylated proteins [21-23]. For biological function, however, SHP1 plays a dominant negative regulation role in the pathway $[24,25]$ while SHP2 plays a major positive role [26-28]. Phosphorylation of SHP2 activates associate proteins such as Grb2 and Gab2 and form a protein complex SHP2/Grb2/Gab2. This complex then activates the downstream target RAS and other components of the RAS/ERK pathways [29-31]. SHP2 is encoded by PTPN11, a proto-oncogene in hematologic cells [32]. Mutation of PTHN11 has been associated with juvenile myelomonocytic leukemias, neuroblastoma, melanoma, acute myeloid leukemia, breast cancer, lung cancer, and colorectal cancer [33]. SHP2 protein levels are elevated in some cancers including cervical cancer [34] and approx $72 \%$ of breast cancer cell lines [35]. Many cell types express SHP2 however, SHP1 is expressed in a restricted number of cell types [36, 37]. These data indicate that compared with SHP1, SHP2 is more likely to be an onco-protein involved in cancer development. Indeed, several studies have shown that inhibition of SHP2 can retard cancer cell growth [38]. Inhibition of SHP2 gene expression with shRNA was also associated with cell transformation from mesenchymal to epithelial cells, indicating a promoting role of SHP2 in carcinogenesis [39]. A recent study has shown that SHP2 plays an essential role in the initiation, progression, and metastasis of breast cancer by activating stemness-associated transcription factors such as c-Myc and ZEB1 [40], further demonstrating its oncogenic role in cancer stem cells. However, there is no report on if $\gamma$ T3 targets SHP2 in any cancer types.

Breast cancer is the leading cause of cancer related death among women. Though some studies have shown that using $\gamma$-T3 can effectively induce apoptosis or cell cycle arrest in breast cancer cells $[41,18]$, there is no report on $\gamma$-T3 treating breast CSCs. Particularly, there is no report exploring the potential targets of SHP1 and SHP2 in breast CSCs. In this study, we have demonstrated that $\gamma$-T3 had a broad inhibitory effect on human epithelial CSCs including those from breast, colon, and cervical cancers. We found that apart from the effect on SHP1, $\gamma$-T3 also targeted SHP2 in breast cancer and that the $\gamma$-T3 inhibitory effect on CSC growth was through the RAS/ERK pathway. Moreover, we report here that the level of phosphorylated SHP2 protein increases in breast CSCs, compared with their parental cancer cells, suggesting that SPH2 may play an important role in breast CSC growth and may be considered as a potential therapeutic target for breast CSCs.

\section{Methods}

\section{Cell lines and mammosphere culture}

This study did not involve in human subjects including human material or human data. Epithelial cancer cell lines of breast cancer MCF-7 (ATCC, HTB-22 $2^{\text {Tw }}$ ), colon cancer HCT-116 (ATCC, CCL-247 ${ }^{\mathrm{Tm}}$ ), and cervical cancer HeLa (ATCC, CCL-2) were purchased from ATCC (during years 2010-2012) and maintained in complete Dulbecco's Modified Eagles Medium (DMEM, Invitrogen, Australia) as previously described [42]. The sphere culture medium was prepared as previously reported [43]. For the first passage of sphere culture, $1 \times 10^{4}$ cancer cells were seeded into a T25 flask with $6 \mathrm{ml}$ of sphere culture medium. Cells were cultured in suspension for 4 days at $37{ }^{\circ} \mathrm{C}$ with $5 \%$ $\mathrm{CO}_{2}$ and an additional $2.5 \mathrm{ml}$ of sphere culture medium was added to the culture. The culture continued for another 3-4 days and spheres were harvested by centrifugation at $300 \times \mathrm{g}$ for $3 \mathrm{~min}$ and their numbers were counted after re-suspension in $2-5 \mathrm{ml}$ medium. For the second and following passage sphere culture, the spheres were treated with 1:1 diluted $2.5 \%$ TrypsinEDTA (Invitrogen, Australia) for $5 \mathrm{~min}$ at $37{ }^{\circ} \mathrm{C}$ and washed with sphere culture medium. Sphere cells were separated by repeating pipetting. The separated cells were passed through a cell strainer $(40 \mu \mathrm{M}, \mathrm{BD}$, Australia) and were counted for continuous sphere culture or other assays in low-adherence 6-well plates (Sigma-Aldrich, Australia) or T25 flasks.

\section{$\mathrm{Y}$-T3 treatment, flow cytometry, and fluorescence microscopy}

Highly purified $\gamma$-T3 was provided by Davos Life Science Pte. Ltd, Singapore and was prepared as a stock solution in $100 \%$ ethanol at a concentration of $20 \mathrm{mg} / \mathrm{ml}$ and stored at $-20{ }^{\circ} \mathrm{C}$ freezer. The stock solution was diluted in ethanol into $2 \mathrm{mg} / \mathrm{ml}$ and added directly into the sphere cultural medium at different concentrations. The control group was added with same amount of ethanol. The cells were cultured for 7-8 days until spheres formed. For FACS analysis, 
cells were harvested, dispersed from spheres and stained with antibodies to CD44 or CD133 conjugated with FITC and CD24 conjugated with RPE (Invitrogen, Australia) at concentrations of $1: 100\left(\mathrm{~V} / \mathrm{V}, 5 \times 10^{5}\right.$ cells). The cells were washed 2 times with $1 \%$ fetal calf serum (FCS)/PBS then fixed with $2 \%$ paraformaldehyde/PBS for FACS analysis using Calibur or FACS Canto (BD, Australia).

\section{Immuno-blotting}

Immune-blotting analysis was conducted as previously described [42]. The separated cells from mammospheres and cell lysates were prepared in RIPA buffer after $\gamma$-T3 treatment. Before total protein measurement, the samples were sonicated briefly. Anti-human $\beta$-tubulin antibody was from Sigma-Aldrich. Anti-pERK antibody was from Cell signaling. Rabbit anti-SHP2 antibody was from Sigma-Aldrich and rabbit anti-SHP2 (pS576) polyclonal antibody was from Invitrogen USA. Mouse antiSHP1 antibody was purchased from Cell Signaling Technology.

\section{Real-time RT-PCR}

Total RNA extraction from mammosphere cells was prepared as instructed by the manufacturer using TRIzol $^{\circ}$ reagent (Invitrogen, Australia). Reverse transcription reactions were performed with oligo-dT primer using the High Capacity cDNA RT Kit (Applied Biosystems). Real-time PCR was carried out with SYBR green master mixture (Promega) on a Rotor-Gene RG-3000 (Corbett Research, Australia) with the program pre-heating $95^{\circ} \mathrm{C} 10 \mathrm{~min}$; then 40 cycles of $94{ }^{\circ} \mathrm{C} 15 \mathrm{~s} ; 58{ }^{\circ} \mathrm{C} 30 \mathrm{~s}$; and $72{ }^{\circ} \mathrm{C} 45 \mathrm{~s}$. The primers were: TGF- $\beta-1$ : F $5^{\prime}$-CAACAATTCCTGGCGATACC, R 5 '-GAACCCG TTGATGTCCACTT; TGF- $\beta$-2: F 5' -GAGTGCCTGAACAACGGATT, R 5' -TGCAGCAGGGACA GTGTAAG; TGF- $\beta$-3: F 5 '-GCAACTTGGAGGAGAACTGC, R 5'-CTGTGGGTTGTGTCTGC ACT; LIF: F 5' -CCCTGTCGCTCTCTAAGCAC, R 5' -ATCCTGGACAAGGGTGAGTG; H-Ras: F 5'-GTGGTCATTGATGGGGAGAC, R 5'-ACGTCATCCGAGTCCTTCAC; K-Ras: F 5'-TGT CAAGCTCAGCACAATCTG, R 5'-GGTAGGGAGGCAAGATGACA; ERBB2: F 5'-GACATTGACGAGACAGAG, R 5'-ACACAGTCACACCATAAC; ESR1: F 5'-CACATCAGGCACATGAGTAACAA, R 5'-TCCAGC AGCAGGTCATAGAG; SHP1: F 5' TTTCAAGAAGA CGGGGATTG, R 5' CGGACTCCTGCTTCTTGTTC; SHP2: F 5' AGAGCCACCCTGGAGATTTT, R 5' CT CCTCCACCAACGTCGTAT. The internal control was $18 \mathrm{~S}$ rRNA (F: 5'-CCATCGAACGTCTGCCCTA; R: 5'-TC ACCCGTGGTCACCATG) is used to normalize target gene expression.
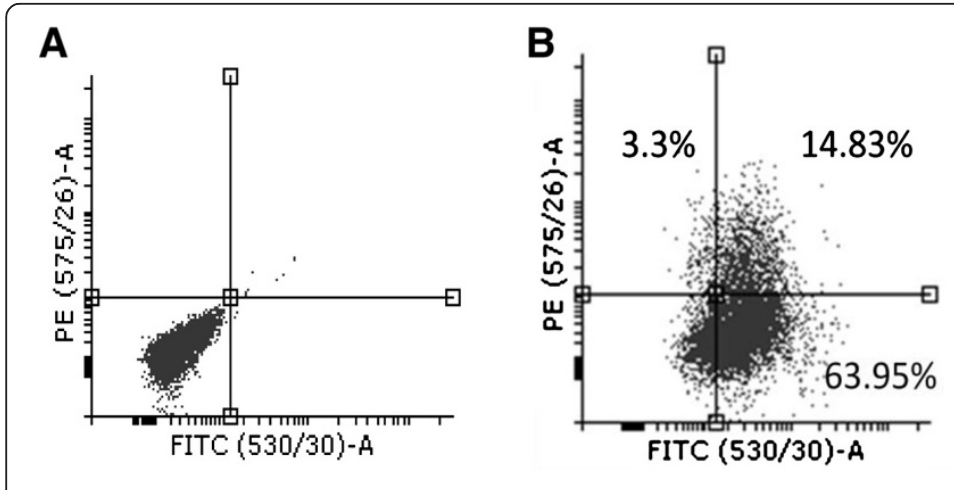

D

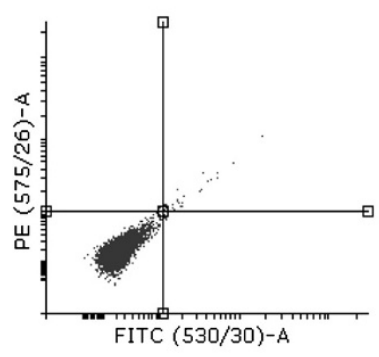

E

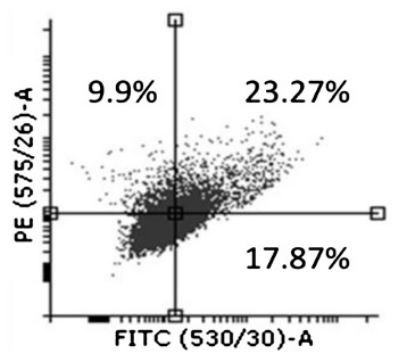

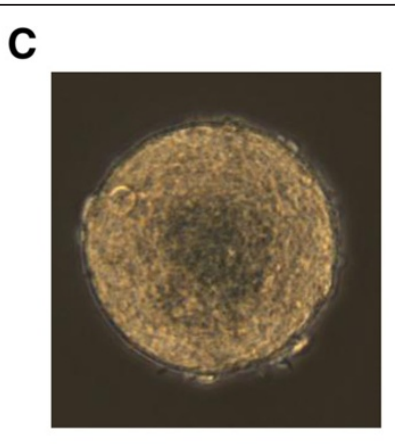

$\mathbf{F}$

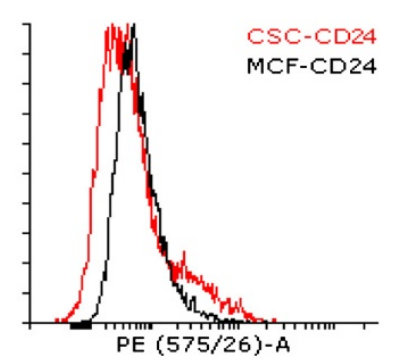

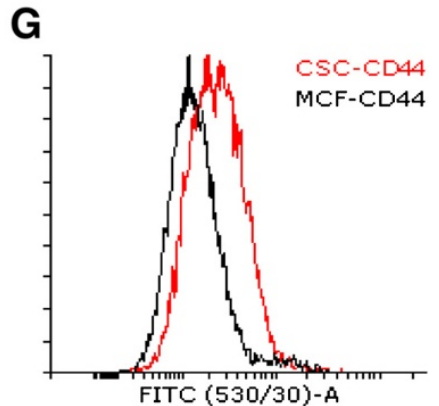

Fig. 1 CD44 and CD24 expressions of mammosphere and MCF-7 cells: Mammosphere cells and their parental MCF-7 cells were labeled for CD44 and CD24 expression. $\mathbf{a}$ antibody isotype controls of mammosphere cells. $\mathbf{b}$ mammosphere cells stained with CD44 and CD24 antibodies conjugated with FITC and PE respectively. $\mathbf{c}$ an image of a mammosphere cultured for 8 days, the compacted sphere contains numerous cells. $\mathbf{d}$ MCF-7 cells stained with isotype controls. e MCF-7 cells stained with CD44 and CD24 antibodies conjugated with FITC and PE respectively. $\mathbf{f}$ and $\mathbf{g}$ histogram diagrams to show the CD24 (f) and CD44 (g) expression shifts (the decrease of CD24 and the increase of CD44 in mammosphere cells) 


\section{Data analysis}

Besides technical repeats (3 repeats) of real-time RTPCR, biological repeats (3 times) were performed. Data collected from each (experimental and control) group were expressed as mean $\pm \mathrm{SD}$. The one-way ANOVA and unpaired $t$-test (GraphPad Prism 6 program) were used to analyze the differences between groups and discriminate the significant differences (two-tails, $P<0.05$ ) between experimental and control groups.

\section{Results}

\section{Mammosphere cells and treatment with $\mathrm{\gamma}$-T3}

We used the reported sphere culture method $[43,44]$ to culture mammospheres from breast carcinoma cell line MCF-7. The mammosphere formation rate was about $20 \%$ from the cell line in several generations (passages) of culture. The mammosphere cells were separated and characterized for surface expression of CD44 and CD24, which are commonly considered as markers of breast cancer stem cells [2]. The results showed CD44 expression increased in mammosphere cells and the positive cell proportion increased from $5.62 \%$ of parent MCF-7 cells to $45.65 \%$ of mammosphere cells (Fig. 1). Meanwhile, the expression of CD24 alone and CD24/CD44 double positive cells was deceased from 15.58 to $5.99 \%$ and from 18.99 to $12.18 \%$, respectively (Fig. 1). These profiles suggest that after sphere culture the mammosphere cells are enriched for breast cancer stem-like cells, which is consistent with previous reports [2, 45]. We then treated mammosphere cells with $\gamma$-T3 in sphere culture by adding $\gamma$-T3 directly into the sphere culture medium. As shown in Fig. 2, $\gamma$-T3 exhibited a dose-dependent inhibition of the growth of mammosphere cells and sphere formation from MCF-7 cells. At $5 \mu \mathrm{g} / \mathrm{ml}$, the sphere growth was totally inhibited (Fig. 2a).
A

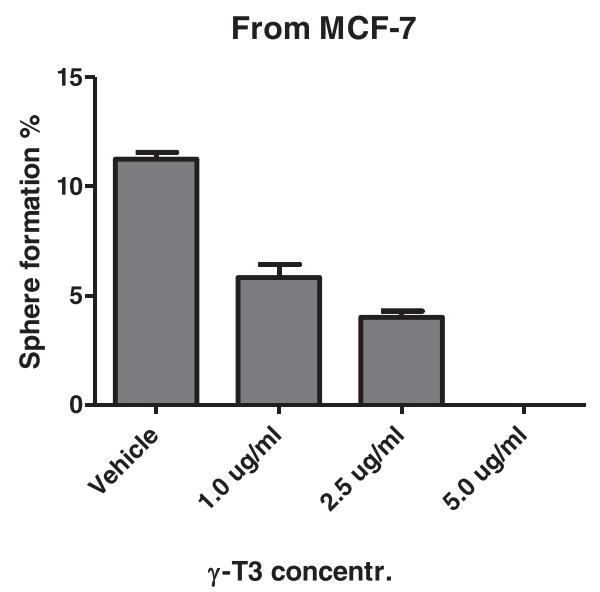

C From HCT-116

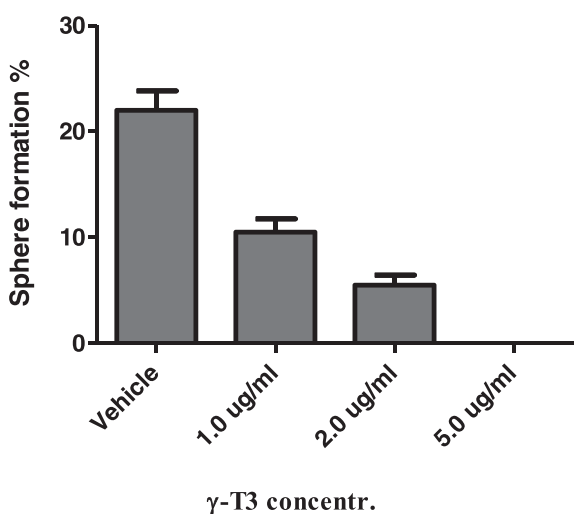

B

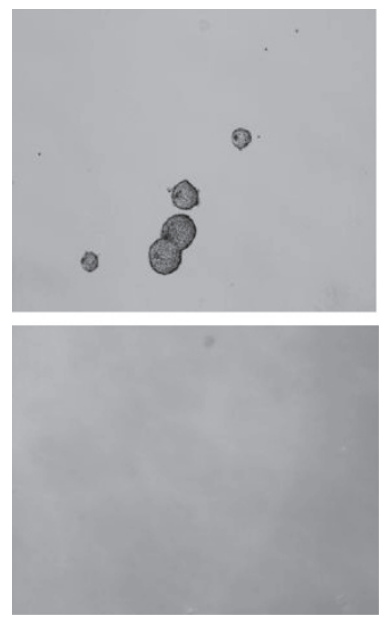

D From HeLa

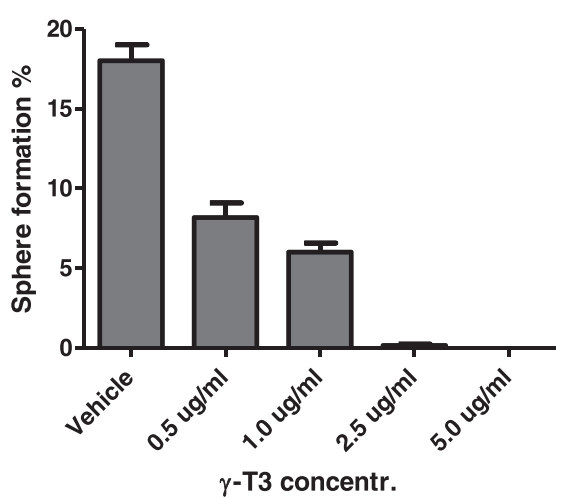

Fig. 2 Dose-dependent inhibition of sphere growth of three epithelial cancers by $\gamma$-T3: a $\gamma$-T3 inhibited mammosphere formation and growth from MCF-7 cells in a dose-dependent manner from 1.0 to $5.0 \mu \mathrm{g} / \mathrm{ml}$. b morphology of mammosphere in vehicle control and in 5 $\mu \mathrm{g} / \mathrm{ml} \mathrm{G}-\mathrm{T} 3$ treatment. $\mathbf{c}$ and $\mathbf{d}$ In addition to breast cancer, $\mathrm{Y}$-T3 inhibited sphere formation of colon (HCT-116) and cervical (HeLa) cancers respectively 


\section{Y-T3 inhibits spherical cell growth of other epithelial cancers}

To extend the above observations in breast cancer to other epithelial cancers, we isolated sphere forming cells (SFCs) from cervical (HeLa cells) and colon (HCT-116 cells) cancers using the same sphere culture method and analyzed their surface markers. The results showed that these SFCs expressed specific markers as expected (Additional file 1: Figure S1) which were consistent with previous reports [44, 46], suggesting that after sphere culture, CSCs are enriched in both cancer cell lines. We treated these spherical cells with $\gamma$-T3. Similar growth inhibition to mammospheres was observed for these two cancers (Fig. 2c and d), revealing that $\gamma$-T3 has a broad spectrum of inhibition effect on the growth of CSCs in epithelial cancers, including prostate cancer as reported previously [19]. In addition, at a dose of $5.0 \mu \mathrm{g} / \mathrm{ml}$ the growth of CSCs was totally inhibited (Fig. 2). It is also noted that comparisons between the three epithelial cancers showed, cervical CSCs from HeLa cells were slightly more sensitive to $\gamma$ T3 than CSCs from MCF-7 and HCT-116 (Fig. 2).

\section{$\mathrm{Y}$-T3 targets SHP2 and also increases SHP1 protein levels in mammosphere cells}

To test whether SHP1 and/or SHP2 are affected by $\gamma$-T3 treatment, we firstly measured mRNA levels of SHP1 and SHP2 in $\gamma$-T3 treated mammosphere cells. SHP1 gene expression did not increase in a dose dependent way as $\gamma$-T3 dose increased (Fig. 3a). But as we expected, SHP2 gene expression did decrease in a dose dependent manner (Fig. 3b). To confirm this in protein levels, we measured total protein levels of SHP1 and SHP2 in $\gamma$-T3 treated mammosphere cells. As shown in Fig. 3c and d, SHP1 levels increased while SHP2 levels decreased as $\gamma$ T3 dose increased. To further prove that SHP2 biological function was inhibited by $\gamma$-T3 treatment, we also measured phosphorylated SHP2 protein (p-SHP2) in $\gamma$ -

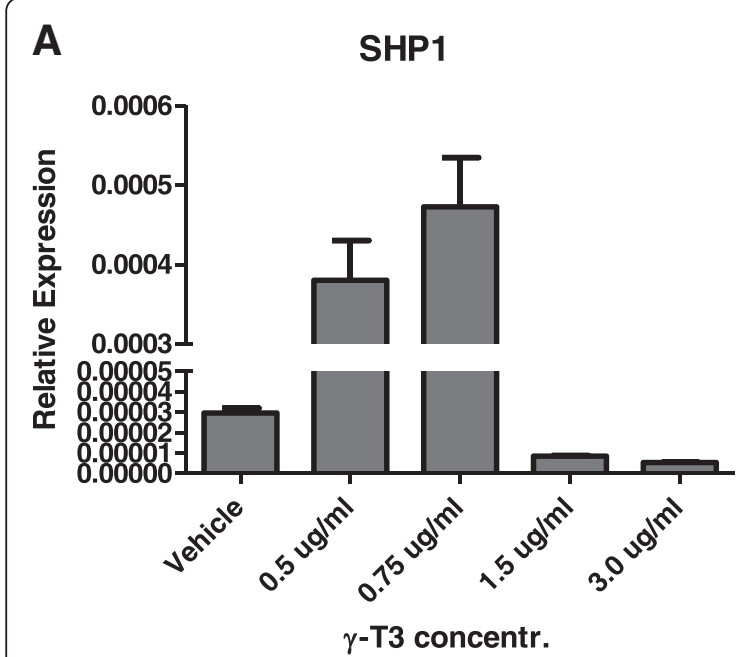

C

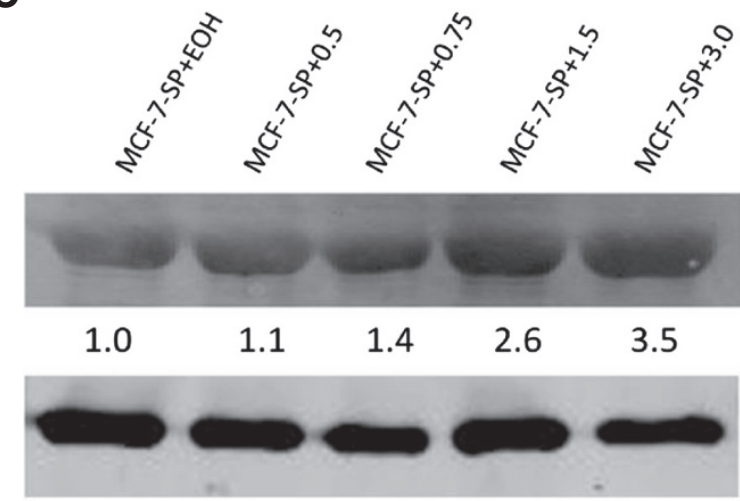

B

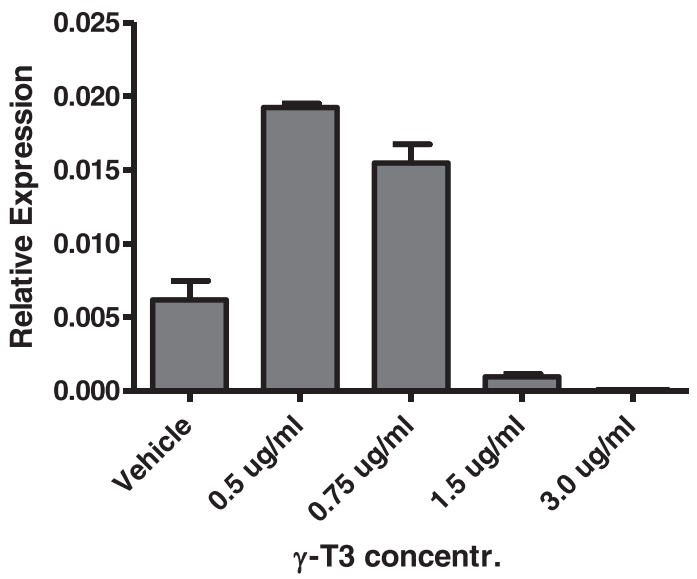

D

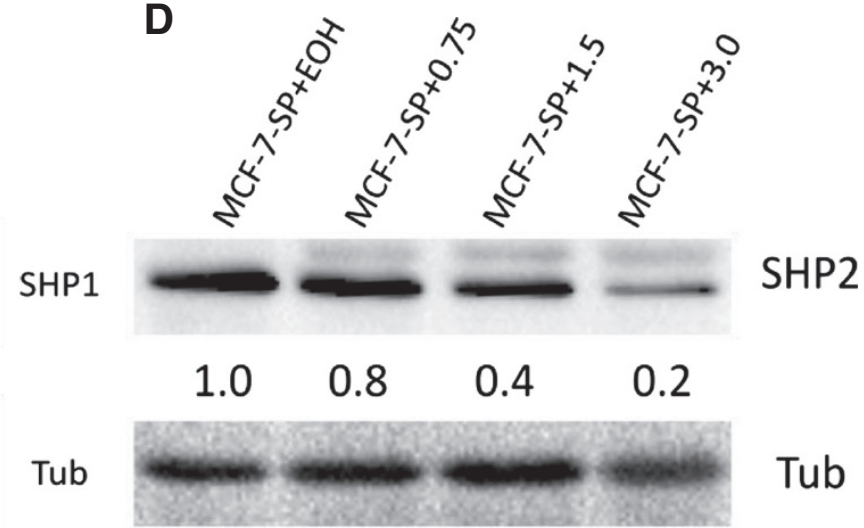

Fig. 3 SHP 1 and SHP2 gene and protein expressions after $\gamma$-T3 treatment. a and $\mathbf{b}$ real-time PCR results showing SHP1 and SHP2 mRNA expression in mammosphere cells treated with different doses of $\gamma$-T3. $\mathbf{c}$ and $\mathbf{d}$ Western blotting results showing SHP1 and SHP2 protein expression levels after $\gamma$-T3 treatment at different doses. EOH ethanol, SP sphere cells, Tub human $\beta$-tublin 
T3 treated cells, the result showed that p-SHP2 also decreased as $\gamma$-T3 dose increased (Fig. 4a). To further explore other possible targets of $\gamma$-T3, we measured the expression of other two closely related genes, ERBB2 and ESR1. ERBB2 is also known as HER 2, and is a trans-membrane receptor protein and an up-stream activator of SHP2 and RAS-ERK pathway [47]. ESR1 is an estrogen receptor which can be associated with the cell surface membrane and after activation can be involved in the activation of MAPK [48]. However, our data indicated that these two gene expressions were not affected by $\gamma$-T3 treatment (Fig. 4b and c). Therefore, we focused on SHP2 protein in this study.

\section{Dose-dependent down-regulation of RAS/ERK pathway by} $\mathrm{Y}$-T3

SH2-domain containing proteins, like SHP2, when phosphorylated on tyrosine residues, culminate in the activation of a number of intracellular signalling cascades including the STAT5, Ras/mitogen activated protein kinase (MAPK) and phosphoinositide-3 kinase (PI3K) pathways [49]. In this singling pathway Ras is a direct downstream component of SHP2, hence we assumed that the down-regulation of SHP2 would also affect Ras gene expression. In addition, the increased SHP1 protein expression by $\gamma$-T3 could also affect RAS/ERK pathway through negatively regulating JAK/Stat pathway. We thus measured Ras gene expression in $\gamma$-T3 treated cells. As we expected, both $\mathrm{H}$-Ras and $\mathrm{K}$-Ras gene expressions decreased as $\gamma$-T3 doses increased (Fig. 5a and b). To further testify that $\gamma$-T3 targeted SHP1 and SHP2 and through RAS/ERK pathway, we also measured another downstream component, the phosphorylated ERK protein in $\gamma$-T3 treated cells and we found that phosphorylated ERK decreased in $\gamma$-T3 treated cells in a dosedependent manner (Fig. 5c). All these results together suggest that SHP1, SHP2, and RAS/ERK pathways have been affected by $\gamma$-T3 treatment. To understand if SHP2 is more activated in mammosphere cells, we compared pSHP2 level in MCF-7 and mammosphere cells (Fig. 5d). The result indicated that an increased p-SHP2 was observed in mammosphere cells.

\section{$\gamma$-T3 did not affect self-renewal pathways in breast CSCs}

It has been shown that TGF- $\beta$ and LIF are two important self-renewal genes for stem cells [50, 51]. In cervical cancer, we showed that silencing human papillomavirus oncogenes E6/E7 with shRNA delivered by lentiviral
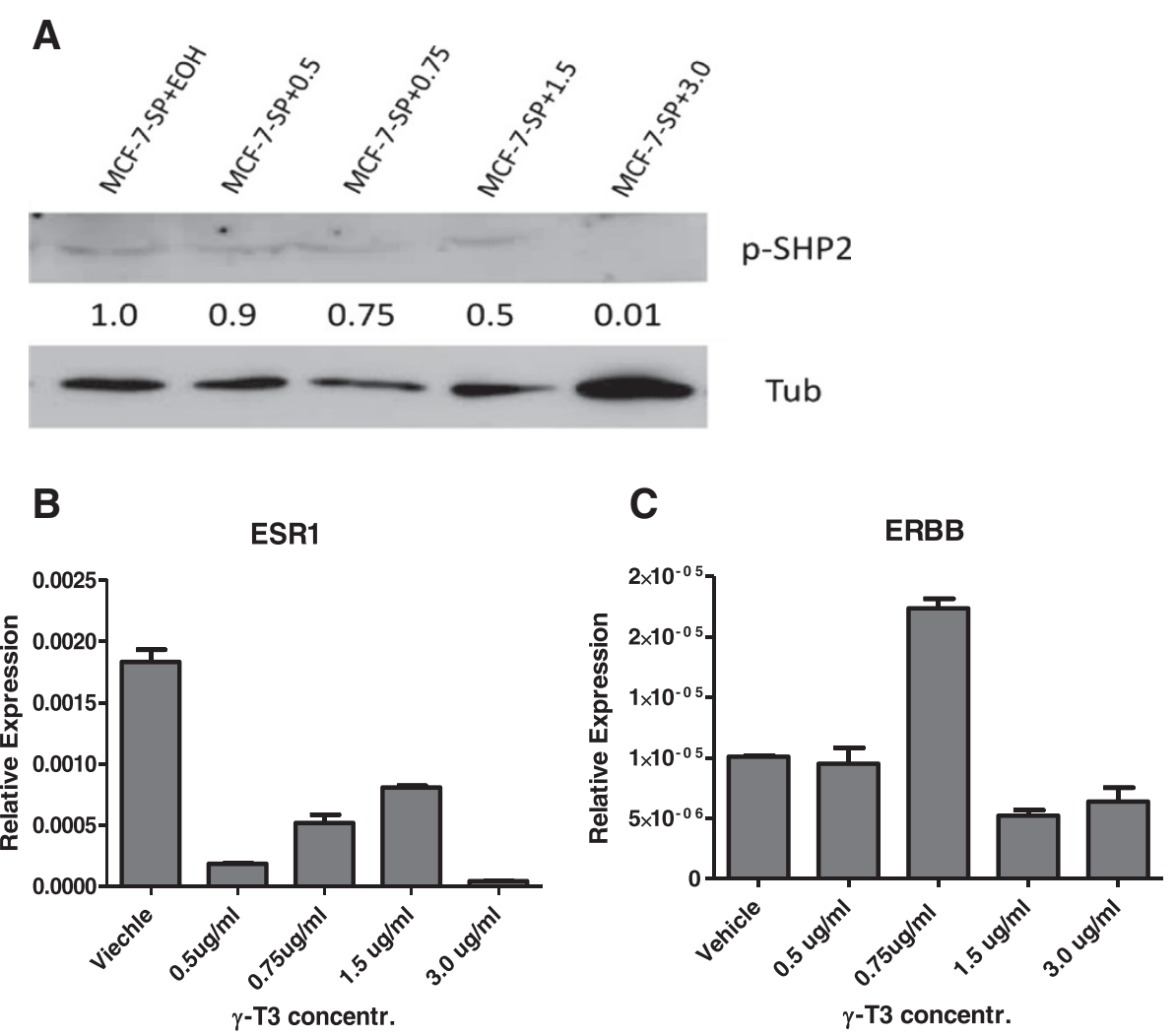

Fig. 4 Phosphorylated SHP2 and other related gene expression after $\gamma$-T3 treatment. a Western blotting result shows phosphorylated SHP2 (p-SHP2) protein levels in mammosphere cells after $\gamma$-T3 treatment with different doses. $\mathbf{b}$ and $\mathbf{c}$, real-time RT-PCR results show mRNA expressions of ERBB and ESR in $\gamma$-T3 treated mammosphere cells. EOH ethanol; SP sphere cells; Tub human $\beta$-tublin 


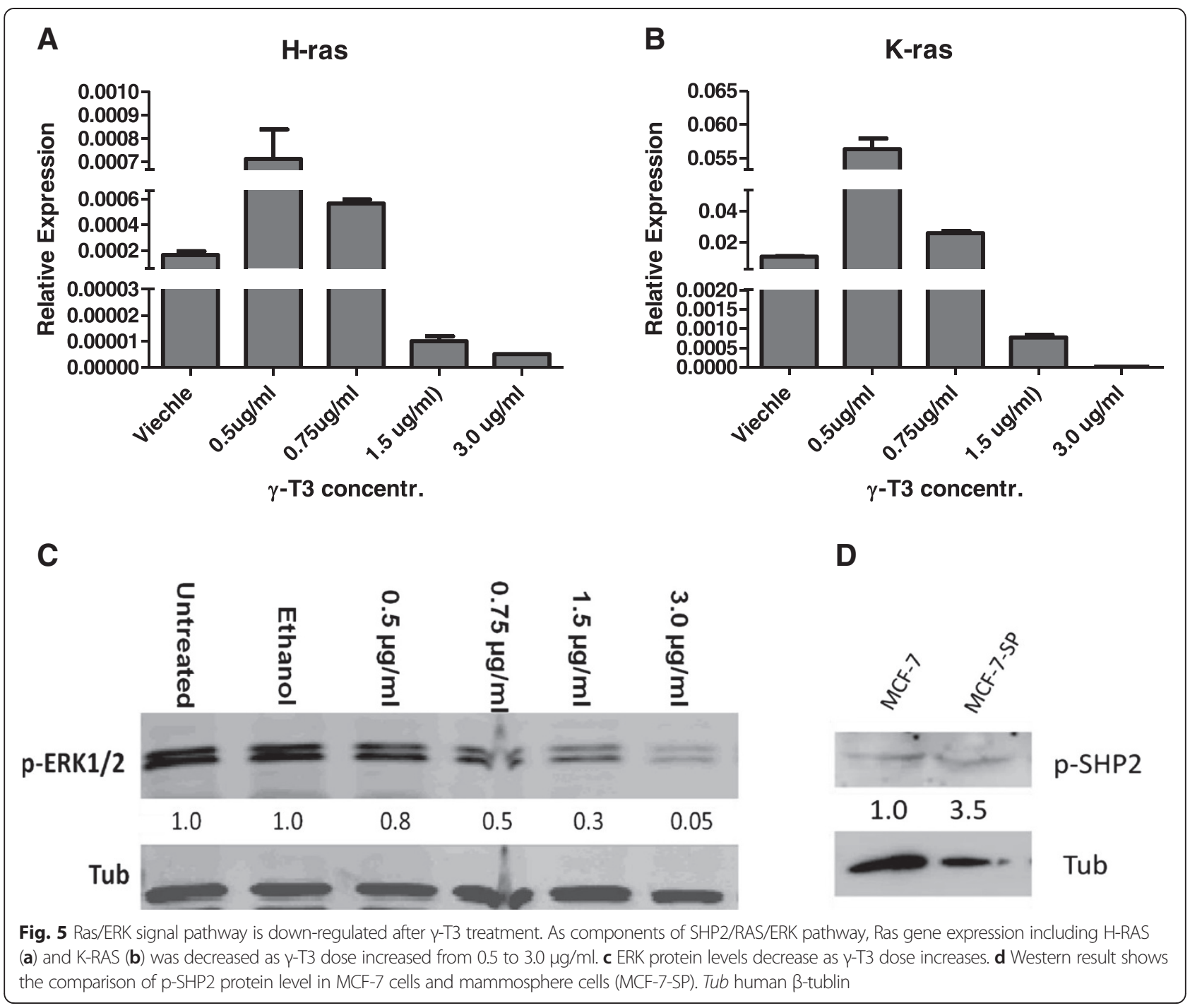

vector in cervical CSCs could lead to the down regulation of self-renewal gene TGF- $\beta$ [44]. To investigate if $\gamma$-T3 also inhibits sphere growth through impairing CSC self-renewal ability by down-regulating TGF- $\beta$ and LIF, we measured gene expression in MCF-7 and mammosphere cells. However, the results failed to show any dose-dependent reduction of gene expressions (Fig. 6). To further confirm this, we also measured gene expression in HeLa sphere cells treated with $\gamma$-T3 (Additional file 1: Figure S2). Similar results were obtained in HeLa sphere cells, suggesting that $\gamma$ T3 inhibits CSC growth was not through inhibiting their self-renewal ability via TGF- $\beta$ and LIF pathways. This result also suggests that $\gamma$-T3 may specifically target SHP1/SHP2/RAS/ERK pathways and which probably leads to the inhibition of C-Myc and CyD transcription factors and anti-apoptosis factor such as Bcl-XL and Spred resulting in cell cycle arrest and apoptosis of mammosphere cells (Fig. 7).

\section{Discussion}

In this study, based on the evidence that $\gamma$-T3 could effectively inhibit sphere growth in three different epithelial cancers, we believed that its target(s) would be vital for self-renewal and proliferation of CSCs and thus tried to identify them. A previous study showed that in skin cancer U266 and SCC4 cells, $\gamma$-T3 inhibited cell growth through induction of SHP1 and suppression of its related JAK/STAT/RAS/ERK signaling pathway [20]. We thought SHP1 could be also a target of $\gamma$-T3 in CSCs. In addition, as SHP2 shares two SH2 domains with SHP1 but has opposite biological functions and is widely expressed in many cell types compared to the restricted expression of SHP1, we thought SHP2 could be a target of $\gamma$-T3 as well. Indeed, our results proved that besides increasing SHP1 protein levels, $\gamma$-T3 also targeted PNPT11/SHP2 and inhibited their expression in mammosphere cells in a dose dependent manner. As a consequence of the dual effects of $\gamma$-T3, the downstream 


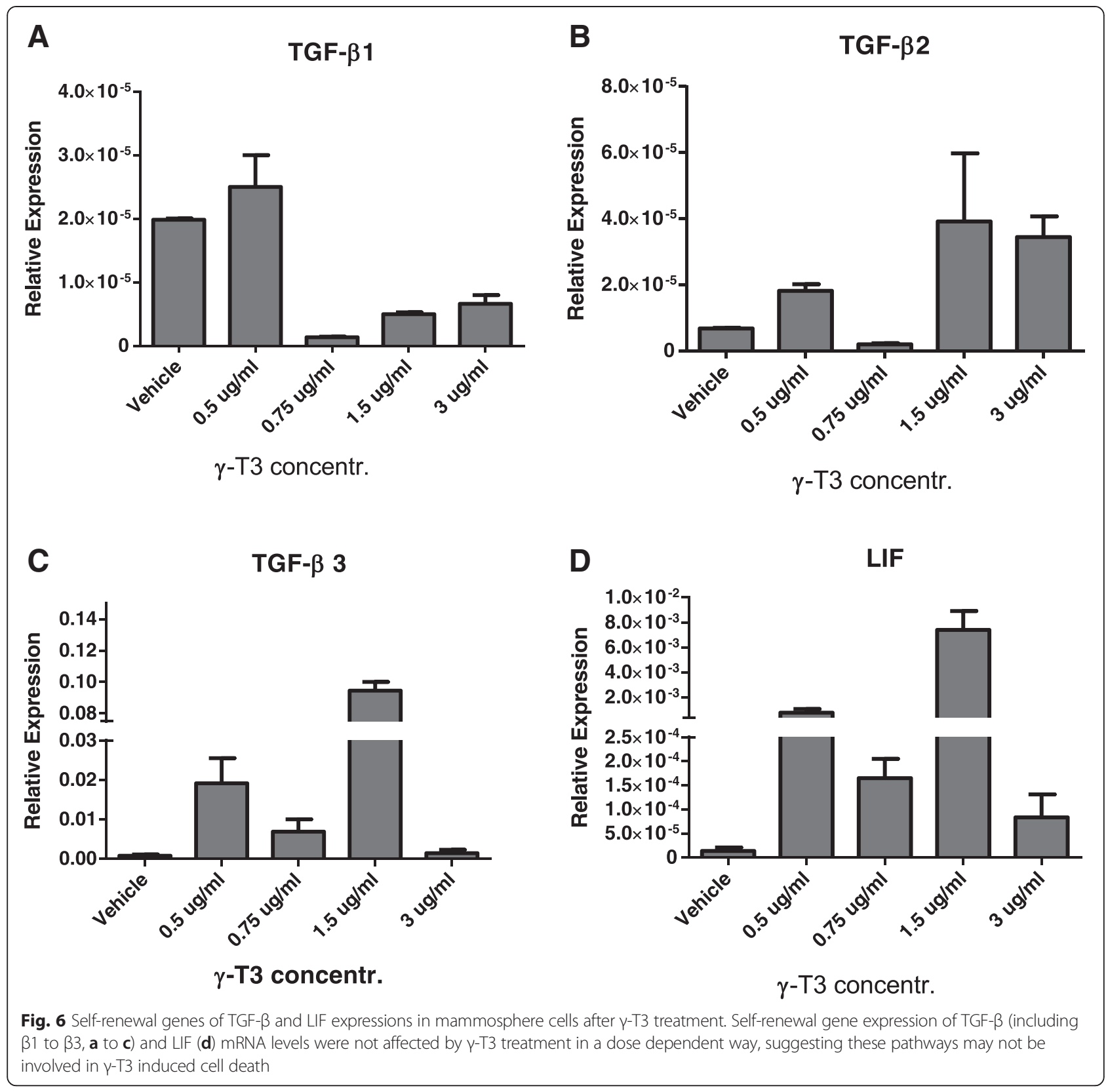

RAS/ERK signal pathway was down-regulated and this further led to the possible cell cycle arrest and apoptosis of mammosphere cells (Fig. 7). In contrast, other unrelated genes/pathways such as ERBB2, ESR1, and TGF- $\beta$ were not affected, indicating that SHP1 and SHP2 are specific targets of $\gamma$-T3 in at least mammosphere cells. As $\gamma$-T3 also effectively inhibits the spherical cell growth of colon and cervical cancers, we assume their SHP1 and 2 had been affected. To our knowledge, the study of the effect of $\gamma$-T3 on both SHP1 and SHP2 in mammosphere cells has not been reported before. Particularly, the down-regulation of PNPT11/SHP2 in mammospheres by $\gamma$-T3 is a new finding. The implication of this study is that SHP2 may serve as a target for breast CSC therapy in the future. Our study also provides evidence that $\gamma$-T3 can be considered as a promising therapeutic candidate to target both SHP1/SHP2 in breast CSCs. As treating breast cancer cells (eg. MCF-7) normally needs a dose ranged from 10 to $40 \mu \mathrm{g} / \mathrm{ml}$, if this dose is applied in clinical settings, it will be sufficient to totally inhibit breast CSC growth according to our present data.

Our results are consistent with a recent study in which the authors demonstrated that SHP2 played a fundamental role in the initiation, progression, and metastasis of human epidermal growth factor receptor 2 (HER2)positive and triple-negative breast cancers [40]. They 


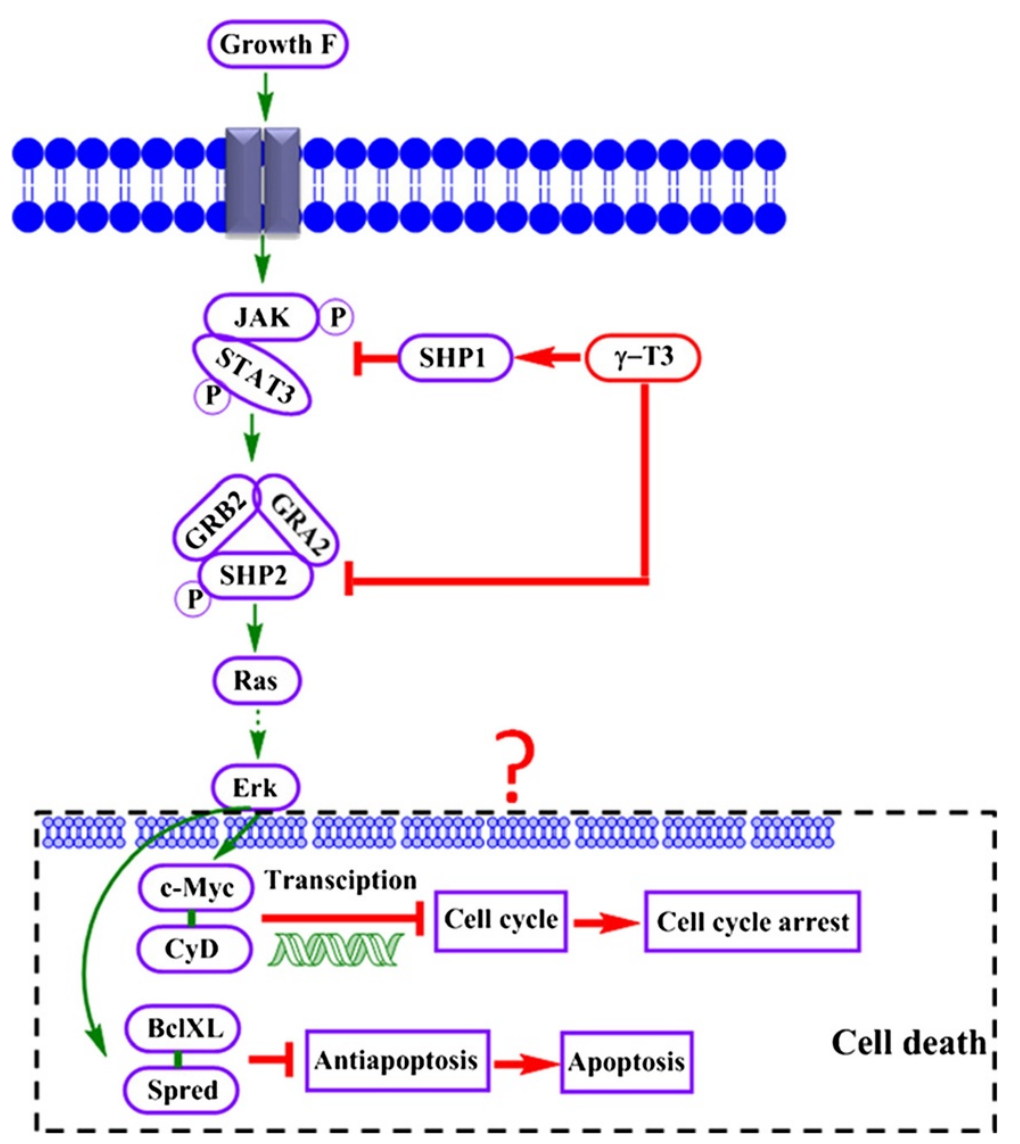

Fig. 7 A schematic diagram to show SHP/RAS/ERK pathway and $\gamma$-T3 targets in mammosphere cells. In mammosphere cells, $\gamma$-T3 targets both SHP1 and SHP2 to down regulate RAS/ERK pathway and subsequently leads to the cell death in mammospheres. For SHP1, $\gamma$-T3 promotes its protein production to down-regulate Ras/ERK pathways. $Y$-T3 through down-regulating the protein level and phosphorylation of SHP2 reduces the interaction with associated proteins such as GRB2 and GAB2, leading to activation of the RAS/ERK pathway. It is postulated that in the nucleus, the double action of down-regulation of ERK interferes with the transcription factors C-myc and Cyd ensuring normal cell cycles (green arrows) and the anti-apoptosis factors Bcl-XL and Spred subsequently cause cell cycle arrest and apoptosis of the mammosphere cells (Red arrows)

showed that knockdown of SHP2 eradicated breast tumour-initiating cells in xenograft models, and SHP2 depletion prevented invasion in three-dimensional cultures and in a transductal invasion assay in vivo. In addition, SHP2 knockdown in established breast tumours blocked their growth and reduced metastasis [40]. They also showed that these effects of SHP2 were through activation of stemness-associated transcription factors, including v-myc myelocytomatosis viral oncogene homolog (c-Myc) and zinc finger E-box binding homeobox 1 (ZEB1). C-Myc is also a downstream component of Ras/ ERK pathway, indicating their result is consistent with our data. In addition, in the study they showed that depletion of SHP2 could impair selfrenewal ability of breast cancer cells by using sphere culture method [40]. This is obviously confirmed by our current study. However, in our study we further demonstrated that this was not through TGF- $\beta$ and LIF pathways (Fig. 6). All data above support our conclusion and further prove that SHP2 provides a rationale target for breast CSC therapy and $\gamma$-T3 should be considered as a good candidate drug for the therapy.

SHP2 is a member of the non-receptor protein-tyrosine phosphatase family and its cell survival, proliferation, migration, and differentiation functions have been well characterized in hematopoietic cells and hematopoietic stem cells (HSCs). The loss of Ptpn11 in murine hematopoietic cells can lead to bone marrow aplasia and lethality [36]. Mutant of Ptpn11 gene in mice show a rapid loss of HSCs and immature progenitors of all hematopoietic lineages in a gene dosage-dependent and cell-autonomous manner. Ptpn11-deficient HSCs and progenitors undergo apoptosis concomitant with increased Noxa expression. Mutant HSCs/progenitors also show defective ERK and Akt activation in response to stem cell factors and diminished thrombopoietin-evoked ERK activation [36]. Overexpression of SHP2 was associated with leukemogenesis in adult human leukemia [52]. Thus, Shp2 plays a critical role in controlling the survival and maintenance of HSCs and immature progenitors in vitro and in vivo. 
SHP2 has been related to oncogenesis because like Src (a well-known oncogene), PTNT11 is also reported as a proto-oncogene [32]. It has been reported that the mutation of PTPN11 is associated with high prevalence of juvenile myelomonocytic leukemias, neuroblastoma, melanoma, acute myeloid leukemia, breast cancer, lung cancer, and colorectal cancer [33]. Moreover, SHP2 protein is elevated in some cancers including cervical cancer which is high associated with HPV infection [34]. In breast cancer, $72 \%$ cancer cell lines have increased amounts of the SHP2 protein and dominant-negative SHP2 blocked the growth of breast cancer cells [35]. Furthermore, overexpression of SHP2 appears to have a positive relationship to HER2 overexpression, nuclear accumulation of hormone receptors, higher tumour grade and lymph node metastasis; which suggests that SHP2 promotes breast oncogenesis [35]. In addition, SHP2 promotes HER2induced signalling and transformation at least in part by dephosphorylating a negative regulatory autophosphorylation site $[35,40]$. However we showed that $\gamma$-T3 induced SHP2 reduction did not affect ERBB2/HER2 at mRNA levels. Further investigations of protein levels are needed. These results suggest that SHP2 might serve as a therapeutic target against breast cancer and other cancers characterized by ERBB2/HER2 overexpression [53]. However, SHP2 and its potential role in CSCs of solid tumors have not been studied or reported much. In this study, we showed that like in HSCs, p-SHP2 was expressed at higher levels in mammosphere cancer cells compared with parental MCF-7 cells, suggesting that it may play an important role in breast CSCs. This observation can be extended to the spherical cells of two other epithelial tumors, colon and cervix, which showed a similar mammosphere inhibition, however further experimental data are needed to prove this.

\section{Conclusion}

Apart from having confirmed the therapeutic value of $\gamma$-T3, this study has also demonstrated that SHP2 is a potential biomarker and therapeutic target for breast CSCs. In addition, it seems that this target may be widely distributed in other epithelial CSCs including colon, cervical and probably prostate cancers. From the therapeutic point of view, $\gamma$-T3 has the potential to be an effective drug to target both differentiated cancer cells and CSCs, which is better than conventional chemotherapeutic drugs that only target differentiated cancer cells.

\section{Additional file}

Additional file 1: Figure S1. Characterization of sphere cell forming cells cultured from colon cancer cell line HCT-116. Figure S2. Self-renewal gene expressions of $\mathrm{Y}$-T3 treated HeLa spherical cells. (DOCX $167 \mathrm{~kb}$ )

\section{Abbreviations}

CSCs: Cancer stem cells; SHP1 and 2: Src homology 2 domain-containing phosphotase 1 and 2; SFCs: Sphere forming cells; TGF- $\beta$ : Beta transformation growth factor; LIF: Leukemia inhibitory factor; ERBB2: V-erb-b2 avian erythroblastic leukemia viral oncogene homolog 2; ESR1: Estrogen receptor.

\section{Competing interests}

The authors declare that there are no competing financial or non-financial interests.

\section{Authors' contributions}

WG, $C Y, Y X, P L$ designed the study, analyzed the data and wrote the paper. WG, IP, MY, FXZ carried out the experiments and help in analyzing the data. All authors had read the manuscript and agreed with the presentation of the manuscript.

\section{Acknowledgement}

The authors would like acknowledge the financial support from Cancer Council Queensland (CCQ) for the project grant to CY and WG and the financial support of Australia Research Council. The authors would also like to thank Dr. Barbara Rolfe for her kind technical support and help in this study.Finally we acknowledge the technical support from the Australian National Fabrication Facility (ANFF).

\section{Author details}

${ }^{1}$ Australian Institute for Bioengineering and Nanotechnology, the University of Queensland, The corner of Cooper Rd. St Lucia, Brisbane QLD 4072, Australia. ${ }^{2}$ Institute of Health and Biomedical Innovation, Queensland University of Technology, Brisbane, Australia. ${ }^{3}$ School of Biomedical Science, the University of Queensland, Brisbane, Australia.

Received: 19 March 2015 Accepted: 21 August 2015

Published online: 28 August 2015

\section{References}

1. Visvader JE, Lindeman GJ. Cancer stem cells in solid tumours: accumulating evidence and unresolved questions. Nat Rev Cancer. 2008;8(10):755-68.

2. Al-Hajj M, Wicha MS, Benito-Hernandez A, Morrison SJ, Clarke MF. Prospective identification of tumorigenic breast cancer cells. Proc Natl Acad Sci U S A. 2003;100(7):3983-8.

3. O'Brien CA, Pollett A, Gallinger S, Dick JE. A human colon cancer cell capable of initiating tumour growth in immunodeficient mice. Nature. 2007;445(7123):106-10.

4. Schatton T, Murphy GF, Frank NY, Yamaura K, Waaga-Gasser AM, Gasser M, et al. Identification of cells initiating human melanomas. Nature. 2008;451(7176):345-9.

5. Chen J, Li Y, Yu TS, McKay RM, Burns DK, Kernie SG, et al. A restricted cell population propagates glioblastoma growth after chemotherapy. Nature. 2012;488(7412):522-6.

6. Driessens G, Beck B, Caauwe A, Simons BD, Blanpain C. Defining the mode of tumour growth by clonal analysis. Nature. 2012;488(7412):527-30.

7. Malanchi I, Santamaria-Martinez A, Susanto E, Peng H, Lehr HA, Delaloye JF, et al. Interactions between cancer stem cells and their niche govern metastatic colonization. Nature. 2012;481(7379):85-9.

8. Nakai E, Park K, Yawata T, Chihara T, Kumazawa A, Nakabayashi H, et al. Enhanced MDR1 expression and chemoresistance of cancer stem cells derived from glioblastoma. Cancer Invest. 2009;27(9):901-8.

9. Lou H, Dean M. Targeted therapy for cancer stem cells: the patched pathway and ABC transporters. Oncogene. 2007;26(9):1357-60.

10. Giancotti FG. Mechanisms governing metastatic dormancy and reactivation. Cell. 2013;155(4):750-64.

11. Yamashita T, Wang XW. Cancer stem cells in the development of liver cancer. J Clin Invest. 2013;123(5):1911-8.

12. Vira D, Basak SK, Veena MS, Wang MB, Batra RK, Srivatsan ES. Cancer stem cells, microRNAs, and therapeutic strategies including natural products. Cancer Metastasis Rev. 2012;31(3-4):733-51.

13. Burnett J, Newman B, Sun D. Targeting cancer stem cells with natural products. Curr Drug Targets. 2012;13(8):1054-64.

14. Efferth T. Stem cells, cancer stem-like cells, and natural products. Planta Med. 2012;78(10):935-42 
15. Rajendran P, Li F, Manu KA, Shanmugam MK, Loo SY, Kumar AP, et al. gamma-Tocotrienol is a novel inhibitor of constitutive and inducible STAT3 signalling pathway in human hepatocellular carcinoma: potential role as an antiproliferative, pro-apoptotic and chemosensitizing agent. Br J Pharmacol. 2011;163(2):283-98.

16. Xu WL, Liu JR, Liu HK, Qi GY, Sun XR, Sun WG, et al. Inhibition of proliferation and induction of apoptosis by gamma-tocotrienol in human colon carcinoma HT-29 cells. Nutrition. 2009;25(5):555-66.

17. Mo H, Elson CE. Apoptosis and cell-cycle arrest in human and murine tumor cells are initiated by isoprenoids. J Nutr. 1999;129(4):804-13.

18. Park SK, Sanders BG, Kline K. Tocotrienols induce apoptosis in breast cancer cell lines via an endoplasmic reticulum stress-dependent increase in extrinsic death receptor signaling. Breast Cancer Res Treat. 2010;124(2):361-75.

19. Luk SU, Yap WN, Chiu YT, Lee DT, Ma S, Lee TK, et al. Gamma-tocotrienol as an effective agent in targeting prostate cancer stem cell-like population. Int J Cancer. 2011;128(9):2182-91.

20. Kannappan R, Yadav VR, Aggarwal BB. gamma-Tocotrienol but not gamma-tocopherol blocks STAT3 cell signaling pathway through induction of protein-tyrosine phosphatase SHP-1 and sensitizes tumor cells to chemotherapeutic agents. J Biol Chem. 2010;285(43):33520-8.

21. Bone H, Dechert U, Jirik F, Schrader JW, Welham MJ. SHP1 and SHP2 protein-tyrosine phosphatases associate with betac after interleukin-3 -induced receptor tyrosine phosphorylation. Identification of potential binding sites and substrates. J Biol Chem. 1997;272(22):14470-6.

22. Yu Z, Su L, Hoglinger O, Jaramillo ML, Banville D, Shen SH. SHP-1 associates with both platelet-derived growth factor receptor and the p85 subunit of phosphatidylinositol 3-kinase. J Biol Chem. 1998;273(6):3687-94.

23. Streuli M. Protein tyrosine phosphatases in signaling. Curr Opin Cell Biol. 1996:8(2):182-8.

24. Haque SJ, Harbor P, Tabrizi M, Yi T, Williams BR. Protein-tyrosine phosphatase Shp-1 is a negative regulator of IL-4- and IL-13-dependent signal transduction. J Biol Chem. 1998;273(51):33893-6.

25. Dong Q, Siminovitch KA, Fialkow L, Fukushima T, Downey GP. Negative regulation of myeloid cell proliferation and function by the $\mathrm{SH} 2$ domain-containing tyrosine phosphatase-1. J Immunol. 1999;162(6):3220-30.

26. Frearson JA, Alexander DR. The phosphotyrosine phosphatase SHP-2 participates in a multimeric signaling complex and regulates $T$ cell receptor (TCR) coupling to the Ras/mitogen-activated protein kinase (MAPK) pathway in Jurkat T cells. J Exp Med. 1998;187(9):1417-26.

27. Pazdrak K, Adachi T, Alam R. Src homology 2 protein tyrosine phosphatase (SHPTP2)/Src homology 2 phosphatase 2 (SHP2) tyrosine phosphatase is a positive regulator of the interleukin 5 receptor signal transduction pathways leading to the prolongation of eosinophil survival. J Exp Med. 1997;186(4):561-8

28. Yamauchi K, Milarski KL, Saltiel AR, Pessin JE. Protein-tyrosine-phosphatase SHPTP2 is a required positive effector for insulin downstream signaling. Proc Natl Acad Sci U S A. 1995;92(3):664-8.

29. Cunnick JM, Dorsey JF, Munoz-Antonia T, Mei L, Wu J. Requirement of SHP2 binding to Grb2-associated binder-1 for mitogen-activated protein kinase activation in response to lysophosphatidic acid and epidermal growth factor. J Biol Chem. 2000;275(18):13842-8.

30. Cunnick JM, Meng S, Ren Y, Desponts C, Wang HG, Djeu JY, et al. Regulation of the mitogen-activated protein kinase signaling pathway by SHP2. J Biol Chem. 2002;277(11):9498-504.

31. Ren Y, Meng S, Mei L, Zhao ZJ, Jove R, Wu J. Roles of Gab1 and SHP2 in paxillin tyrosine dephosphorylation and Src activation in response to epidermal growth factor. J Biol Chem. 2004;279(9):8497-505.

32. Chan RJ, Feng GS. PTPN11 is the first identified proto-oncogene that encodes a tyrosine phosphatase. Blood. 2007;109(3):862-7.

33. Bentires-Alj M, Paez JG, David FS, Keilhack H, Halmos B, Naoki K, et al. Activating mutations of the noonan syndrome-associated SHP2/PTPN11 gene in human solid tumors and adult acute myelogenous leukemia. Cancer Res. 2004;64(24):8816-20.

34. Tao XH, Shen JG, Pan WL, Dong YE, Meng Q, Honn KV, et al. Significance of SHP-1 and SHP-2 expression in human papillomavirus infected Condyloma acuminatum and cervical cancer. Pathol Oncol Res. 2008;14(4):365-71.

35. Zhou X, Coad J, Ducatman B, Agazie YM. SHP2 is up-regulated in breast cancer cells and in infiltrating ductal carcinoma of the breast, implying its involvement in breast oncogenesis. Histopathology. 2008;53(4):389-402.
36. Chan G, Cheung LS, Yang W, Milyavsky M, Sanders AD, Gu S, et al. Essential role for Ptpn11 in survival of hematopoietic stem and progenitor cells. Blood. 2011;117(16):4253-61.

37. Servidei T, Bhide PG, Huang Z, Moskowitz MA, Harsh G, Reeves SA. The protein tyrosine phosphatase SHP-2 is expressed in glial and neuronal progenitor cells, postmitotic neurons and reactive astrocytes. Neuroscience. 1998;82(2):529-43.

38. Scherr M, Chaturvedi A, Battmer K, Dallmann I, Schultheis B, Ganser A, et al Enhanced sensitivity to inhibition of SHP2, STAT5, and Gab2 expression in chronic myeloid leukemia (CML). Blood. 2006;107(8):3279-87.

39. Zhou XD, Agazie YM. Inhibition of SHP2 leads to mesenchymal to epithelial transition in breast cancer cells. Cell Death Differ. 2008;15(6):988-96.

40. Aceto N, Sausgruber N, Brinkhaus H, Gaidatzis D, Martiny-Baron G, Mazzarol $\mathrm{G}$, et al. Tyrosine phosphatase SHP2 promotes breast cancer progression and maintains tumor-initiating cells via activation of key transcription factors and a positive feedback signaling loop. Nat Med. 2012;18(4):529-37.

41. Hsieh TC, Elangovan S, Wu JM. gamma-Tocotrienol controls proliferation, modulates expression of cell cycle regulatory proteins and up-regulates quinone reductase NQO2 in MCF-7 breast cancer cells. Anticancer Res. 2010;30(7):2869-74.

42. Gu W, Putral L, Hengst K, Minto K, Saunders NA, Leggatt G, et al. Inhibition of cervical cancer cell growth in vitro and in vivo with lentiviral-vector delivered short hairpin RNA targeting human papillomavirus E6 and E7 oncogenes. Cancer Gene Ther. 2006;13(11):1023-32.

43. Dontu G, Abdallah WM, Foley JM, Jackson KW, Clarke MF, Kawamura MJ, et al. In vitro propagation and transcriptional profiling of human mammary stem/progenitor cells. Genes Dev. 2003;17(10):1253-70.

44. Gu W, Yeo E, McMillan N, Yu C. Silencing oncogene expression in cervical cancer stem-like cells inhibits their cell growth and self-renewal ability. Cancer Gene Ther. 2011;18(12):897-905.

45. Yu F, Yao H, Zhu P, Zhang X, Pan Q, Gong C, et al. let-7 regulates self renewal and tumorigenicity of breast cancer cells. Cell. 2007;131(6):1109-23.

46. Tsurumi C, Esser N, Firat E, Gaedicke S, Follo M, Behe M, et al. Non-invasive in vivo imaging of tumor-associated CD133/prominin. PLoS One. 2010;5(12), e15605.

47. Olayioye MA. Update on HER-2 as a target for cancer therapy: intracellular signaling pathways of ErbB2/HER-2 and family members. Breast Cancer Res. 2001;3(6):385-9.

48. Kato $\mathrm{S}$, Endoh H, Masuhiro Y, Kitamoto T, Uchiyama S, Sasaki H, et al. Activation of the estrogen receptor through phosphorylation by mitogen-activated protein kinase. Science. 1995;270(5241):1491-4.

49. Wheadon H, Paling NR, Welham MJ. Molecular interactions of SHP1 and SHP2 in IL-3-signalling. Cell Signal. 2002;14(3):219-29.

50. Singh A, Settleman J. EMT, cancer stem cells and drug resistance: an emerging axis of evil in the war on cancer. Oncogene. 2010;29(34):4741-51.

51. Penuelas S, Anido J, Prieto-Sanchez RM, Folch G, Barba I, Cuartas I, et al. TGF-beta increases glioma-initiating cell self-renewal through the induction of LIF in human glioblastoma. Cancer Cell. 2009;15(4):315-27.

52. Xu R, Yu Y, Zheng S, Zhao X, Dong Q, He Z, et al. Overexpression of Shp2 tyrosine phosphatase is implicated in leukemogenesis in adult human leukemia. Blood. 2005;106(9):3142-9

53. Zhou X, Agazie YM. Molecular mechanism for SHP2 in promoting HER2-induced signaling and transformation. J Biol Chem. 2009;284(18):12226-34.

\section{Submit your next manuscript to BioMed Central and take full advantage of:}

- Convenient online submission

- Thorough peer review

- No space constraints or color figure charges

- Immediate publication on acceptance

- Inclusion in PubMed, CAS, Scopus and Google Scholar

- Research which is freely available for redistribution 